\title{
AMERICAN ORTHOPSYCHIATRIC ASSOCIATION I939 MEMBERSHIP LIST
}

(C) denote's Charter Member; (0), Fellows; $(\mathrm{H})$, Honorary Member. The figures in parenthesis indicate the year of election to membership. Organization names are listed when the business address is given.

Аввот, E. Standley, M.D., 29 Gloucester St., Boston, Mass. (ㄱ) ('29).

Ackerly, Spafford, M.D., Mental Hygiene Clinic, 6ro S. Floyd St., Louisville, Ky. (2) ('3I).

Ackerman, Nathan Ward, M.D., 43 East 78th St., New York, N. Y. ('39).

Ackerson, Luton, Ph.D., Inst. for Juvenile Research, 907 S. Wolcott Ave., Chicago, Ill. (1) ('29). Adams, Walter A., M.D., 3923 Vincennes Ave., Chicago, Ill. (35).

Alexander, Franz, M.D., Institute for Psychoanalysis, 43 E. Ohio St., Chicago, Ill. (1) ('31).

Allen, Mrs. Cornelia D. H., I95 Voorhees Ave., Buffalo, N. Y. (-) ('30).

Allen, Elizabeth, Dept. of Public Welfare, 140 W. Willow St., Syracuse, N. Y. ('29).

Allen, Frederick H., M.D., Child Guidance Clinic, I7I I Fitzwater St., Philadelphia, Pa. (7) (26).

Alpern, Evelyn, M. D., Child Guidance Clinic, Ioo N. Main St., Providence, R. I. ('33).

Amster, Mrs. Fanny, 43-4I Forty-fifth St., Sunnyside, L. I., N. Y. ('39).

Anderson, Forrest N., M.D., Ch. Guidance Clin., I325 W. Adams Blvd., Los Angeles, Cal.. (1) ('29).

Anderson, Harold H., Ph.D., University of Illinois, Urbana, Illinois ('39).

Anderson, Rose G., Ph.D., Psychological Service Center, 522 Fifth Ave., New York, N. Y. ('37).

Anderson, V. V., M.D., The Anderson School, Staatsburg-on-Hudson, N. Y. (I) (C).

Apterar, Herbert H., 33 East 22nd St., New York, N. Y. ('39).

Arxin, Frances S., M.D., 572 Park Ave., New York, N. Y. ('39).

Arrington, Winifred W., Joint Vocational Service, I22 E. 22nd St., New York, N. Y. (1) ('30).

Arthur, Grace, Ph.D., Amherst H. Wilder Ch. Guidance Clin., 279 Rice St., St. Paul, Minn. (6) ("32).

Aten, Eugene L., M.D., Dallas Child Guidance Clinic, 3609 Cedar Springs Rd., Dallas, Tex. ('38).

August, Harry E., M.D., 538 Maccabees Bldg., Detroit, Mich. ('33).

Baxer, Amos T., M.D., P.O. Box G, Briarcliff Manor, N. Y. (i) ('25).

Barbee, Margaret, Henry Watson Children's Aid Society, 204 Lanvale St., Baltimore, Md. ('33).

Bartelme, Phyllis, Ph.D., 990 E. Valley Rd., Santa Barbara, Cal. (29) ('29).

Bartemejer, Leo H., M.D., 8-259 General Motors Bldg., Detroit, Mich. (1) ('29).

Bassett, Clara, Nat. Committee for Mental Hygiene, so W. $50 t h$ St., New York, N. Y. (1) ('28).

BEck, Samuel J., Ph.D., Michael Reese Hospital, 2oth St. and Ellis Ave., Chicago, Ill. (1) ('29).

BEeley, Arthur L., Ph.D., University of Utah, Salt Lake City, Utah ('37).

Bender, Lauretta, M.D., 324 E. 41st St., New York, N. Y. ('37).

Benton, Arthur L., Ph.D., Student Personnel Dept., C. C. N. Y., Convent Ave. and r39th St., New York, N. Y. ('38).

Blanchard, Myron B., Edenwald School for Boys, 225th St. and Boston Rd., Bronx, N. Y. ('34).

Blanchard, Phyllis, Ph.D., Child Guidance Clinic, 17 I I Fitzwater St., Philadelphia, Pa. (J) ('30).

Blau, Abram, M.D., 1075 Park Ave., New York, N. Y. ('39).

Bradiey, Charles, M. D., Emma Pendleton Bradley Home, E. Providence, R. I. ('36).

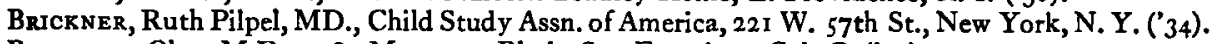

Bridgman, Olga, M.D., I 380 Monterey Blvd., San Francisco, Cal. (E) ('25).

Bridgman, Ralph P., i 26 Old Post Rd., Croton, N. Y. (1) ('31).

Brinker, Dorothy, The Jefferson, Apt. 309, Jefferson and Third Sts., Niagara Falls, N. Y. ('32).

Brockbank, Thomas W., M.D., Catholic Charities Guidance Inst., 181 E. 68th St., New York, N.Y. (2) ('33).

Bronner, Augusta F., Ph.D., Judge Baker Guidance Center, $38 \frac{1}{3}$ Beacon St., Boston, Mass. (1) ('25).

Brown, Frederick W., 69 Cambridge Ave., Garden City, N. Y. ('28).

Brown, Muriel W., Ph.D., Board of Education Bldg., Tulsa, Okla. (' 30 ).

Burling, Temple, M.D., 605 E. Saddle River Rd., Ridgewood, N. J. (D) ('33).

Burlingham, Susan, 218 W. Rittenhouse St., Germantown, Pa. ('39).

Cameron, Eugenia S., M.D., 4I4 E. Lake Ave., Baltimore, Md. ('39).

Challman, S. A., M.D., 67-Io9 Dartmouth St., Forest Hills, L. I., N. Y. ('35).

Chamberlain, Herbert E., M.D., Dept. of Social Welfare, 616 K St., Sacramento, Cal. (25) ('27).

Chapman, Ross McC., M.D., Sheppard and Enoch Pratt Hospital, Towson, Md. (28).

Chasseld, Joseph, M.D., Bennington College, Bennington, Vt. ('38).

Childers, Asher T., M.D., 1721 Berkley Ave., Cincinnati, O. (3) ('31). 
Chornyax, John, M.D., Juvenile Court, 3333 Forbes St., Pittsburgh, Pa. ('38).

CHov, Li-chiu, Dept. Sociology, Ginling College, Chingtu, West China ('36).

Clark, Mary Augusta, Bedford, N. Y. (1) ('3o).

Clirton, Eleanor, 4I Fifth Ave., New York, N. Y. ('27).

Cochran, Mrs. Mary Lue, Livingston, Tex. ('31).

Coghill, Harvie DeJ., M.D., Children's Memorial Clinic, Ioor E. Clay St., Richmond, Va. (1) ('28)

Coleman, Jules Victor, M.D., Hebrew Sheltering Guardian Society, Pleasantville, N. Y. ('39).

Corson, Harold F., M.D., Austen Riggs Foundation, Stockbridge, Mass. ('3I).

Covcr, Mildred Warden, M.D., Cromwell Hall, Cromwell, Conn. (' 31 ).

Crane, Harry W., Ph.D., Box 809, Chapel Hill, N. C. ('37).

Crowell, Sarah Eyre, $194^{2}$ Chestnut St., Harrisburg, Pa. ('36).

Cunningham, James M., M.D., Bur. of Ment. Hygiene, Dept. of Health, Hartford, Conn. (-) ('33).

Dawley, Almena, Child Guidance Clinic, 17 I I Fitzwater St., Philadelphia, Pa. (A) ('3I).

deBerry, Ellett M., M.D., University of Minnesota, Minneapolis, Minn. (E) ('29).

Depres, Marian A., Ph.D., 5525 S. Blackstone Ave., Chicago, Ill. ('39).

Dicks, Grace H., Cleveland Children's Bureau, Ioo Huron Rd., Cleveland, O. ('37).

Dols, Edgar A., Ph.D., The Training School, Vineland, N. J. (5) ('25).

DoмвEY, Edith H., I 5602 Lake Ave., Lakewood, O. ('34).

Doody, William M., M.D., 19 Bentley Ave., Jersey City, N. J. ('35).

Drayer, Calvin Searle, M.D., 4045 Baltimore Ave., Philadelphia, Pa. ('38).

Dummer, Mrs. W. F., 679 N. Michigan Ave., Chicago, Ill. (H) ('26).

Ebavgh, Franklin G., M.D., 1788 Glencoe St., Denver, Colo. (P) ('29).

EIsler, Edwin R., M.D., 43 E. Ohio St., Chicago, Ill. (1) ('29).

Elkind, Henry B., M.D., Mass. Society for Mental Hygiene, 3 Joy St., Boston, Mass. (2) ('28).

Emery, E. Van Norman, M.D., 7390 Norwood Ave., St. Louis, Mo. (E) ('31).

Englander, Charles, M.D., 4I Hillside Ave., Newark, N. J. ('3I).

Ephrow, Harmon S., M.D., 315 Central Park West, New York, N. Y. ('38).

Falstein, Eugene I., M.D., 1255 North State Pkwy,. Chicago, Ill. ('39).

Feder, Leah, Ph.D., Washington University, St. Louis, Mo. ('39).

Fenlason, Mrs. L. S., University of Minnesota, 8 Jones Hall, Minneapolis, Minn. ('37).

Fenton, Norman, Ph.D., Bureau of Juvenile Research, Stanford University, Cal. ('37).

Ferguson, William G., M.D., Child Guidance Clinic, 650 Eleventh St., Niagara Falls, N. Y. ('30).

Finley, Malcolm H., M.D., Skokie School, Winnetka, Ill. ('37).

Fitz-Simons, Marian J., Ph.D., 1737 Burns Ave., Detroit, Mich. ('37).

Foster, Sybil, Child Welfare League of America, r 30 E. 22nd St., New York, N. Y. ('30).

Francis, Mrs. Blythe White, 248 W. 43 rd St., Indianapolis, Ind. (' 37 ).

Frank, Lawrence K., Josiah Macy, Jr., Foundation, 565 Park Ave., New York, N. Y. (1) ('32).

Frankenstein, Greta, M.D., 6i E. 86th St., New York, N. Y. ('39).

FreEman, Rowland G., Jr., M.D., Judge Baker Guidance Center, 381 Beacon St., Boston, Mass. ('39)

FreNCH, Mrs. Lois Meredith, 98 Alexander Ave., Nutley, N. J. (3) ('33).

Fries, Margaret E., M.D., 2I W. 86th St., New York, N. Y. ('36).

Fry, Clem C., M.D., Iog College St., Yale Sta., New Haven, Conn. ('31).

Fuller, Farl W., M.D., 8 Mill Rd., Morris Plains, N. J. (R.F.D.) ('3I).

Garretr, Annette, Smith College School for Social Work, Northampton, Mass. (B) (' 31 ).

Gerard, Margaret W., M.D., 5744 Harper Ave., Chicago, Ill. (i) ('3I).

Gese Ll, Arnold, M.D., I85 Edwards St., New Haven, Conn. (7) ('31).

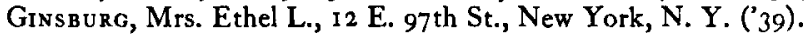

Grre rson, Maxwell, M.D., Institute for Juvenile Research, 907 S. Wolcott Ave., Chicago, Ill. ('36)

Goldman, Julia, Hebrew Sheltering Guardian Society, Pleasantville, N. Y. ('33).

Goldman, Nathan, Lyman School for Boys, Westborough, Mass. ('39).

Gould, Prof. Miriam C., Vassar College, Poughkeepsie, N. Y. ('33).

Green, Rose, Philadelphia Child Guidance Clinic, i7I I Fiztwater St., Philadelphia, Pa. ('38).

Grossman, Grace, National Council of Jewish Women, 285 Schermerhorn St., Brooklyn, N. Y. ('31).

Grossman, Royal G., M.D., 1423 Arthur Ave., Lakewood, O. ('28).

Hagan, Margaret Wood, American Red Cross, St. Elizabeths Hospital, Washington, D. C. ('36).

Hagman, Elmer R., Ph.D., Hartley-Salmon Clinic, 488 Main St., Hartford, Conn. ('33).

Hamilton, E. Louise, Ph.D., Norristown State Hospital, Norristown, Pa. ('39).

Hannett, Frances, M.D., $55^{29}$ Blackstone Ave., Chicago, Ill. ('39).

Hanson, Harold B., M.D., I 891 Portland Ave., St. Paul, Minn. ('39). 
Hardcastle, Douglas Noel, M.D., 2 Lyndhurst Rd., N.W. 3, London, England ('34).

Harrington, Milton, M.D., Napanoch, N. Y. ('32).

Harrison, Frances, 3816 Locust St., Philadelphia, Pa. ('32).

Hartwell, Samuel W., M.D., I 82 Washington Highway, Snyder, N. Y. (1) ('29).

Healy, William, M.D., Judge Baker Guidance Center, $3^{8 \frac{1}{2}}$ Beacon St., Boston, Mass. (D) (C).

Hegge, Thorleif, G., Ph.D., Wayne County Training School, Northville, Mich. (E) ('35).

Heldt, Thomas J., M.D., Henry Ford Hospital, Detroit, Mich. (29).

Helgesson, Uno H., M.D., 66 Paradise Rd., Northampton, Mass. ('31).

Hennessy, Maurice A. R., M.D., Cuyahoga County Juvenile Court, Cleveland, O. ('31).

Hertzman, Jack, M.D., 3149 Harvey Ave., Cincinnati, O. ('39).

Hint, Julia Ford, M.D., Pittsburgh Child Guidance Center, $360_{4}$ Victoria St., Pittsburgh, Pa. ('35).

Hill, Lewis B., M.D., 700 Cathedral St., Baltimore, Md. ('38).

Hincks, Clarence M., M.D., Canadian Nat. Comm. Mental Hygiene, i I St. George St., Toronto, Canada. (1) ('33).

Hincks, Elizabeth M., Ph.D., 58 Washington Ave., Cambridge, Mass. ('32).

Holmer, Paul, M.D., 800 Old Wyomissing Rd., Reading, Pa. ('34).

Horton, Charles B., M.D., Dayton Guidance Center, 225 N. Jefferson St., Dayton, O. ('33).

Igel, Amelia M., 41 W. 54th St., New York, N. Y. ('35).

Irons, Lucia, 43I 3 Marble Hall Rd., Baltimore, Md. ('39).

JAckson, Edith B., M.D., Yale Medical School, New Haven, Conn. ('38).

JACKson, James D., 27 N. Dean Ave., Trenton, N. J. ('30).

JAEger, Martha H., 315 W. 2 Ist St., New York, N. Y. ('33).

JaEnike, R. C. A., M.D., Child Guidance Clinic, Strong Memorial Hospital, Rochester, N. Y. ('33).

Janvier, Carmelite, 3307 Prytania St., New Orleans, La. ('35).

Jefrerson, Roland A., M.D., 208 E. Wisconsin Ave., Milwaukee, Wis. ('33).

Jenkins, Richard L., M.D., State School, Orange County, N. Y. ('39).

Johnson, Eleanor Hope, Ph.D., 99 Sherman St., Hartford, Conn. (i) ('26).

Johnson, George S., M.D., I960 Vallejo St., San Francisco, Cal. (1) ('3o).

Jones, Harold E., Ph.D., Institute of Child Welfare, 2739 Bancroft Way, Berkeley, Cal. ('38).

Joslyn, June J., New Jersey State Hospital, Trenton, N. J. ('34).

Karpman, Benjamin, M.D., St. Elizabeth's Hospital, Washington, D. C. ('39).

Kasanin, Jacob, M.D., Michael Reese Hospital, 2gth St. and Ellis Ave., Chicago, Ill. ( (26).

Kawin, Ethel, 5600 Blackstone Ave., Chicago, Ill. (7) ('32).

Kenworthy, Marion E., M.D., I035 Fifth Ave., New York, N. Y. (F) ('24).

Kimball, Everett, Ph. D., I 86 Elm St., Northampton, Mass. (i) ('30).

KIng, S. Stanley, M.D., Westchester Co. Dept. Ch. Welf., 332 Co. Office Bldg., White Plains, N. Y. (i) ('26).

Kirch Wey, Prof. George W., 435 W. i igth St., New York, N. Y. (H) ('26).

Kirkpatrick, M. E., M.D., 505 City National Bldg., Lansing, Mich. (D) ('3I).

KLopP, Henry Irwin, M.D., Allentown State Hospital, Allentown, Pa. ('38).

KnAPP, Irving, J., M.D., 3370 Altamount Ave., Cleveland Heights, O. ('37).

Kovarsky, Marcel, Jewish Board of Guardians, 228 F. Igth At., New York, N. Y. (1) ('31).

Krugman, Morris, M.D., 228 E. 57th St., New York, N. Y. ('39).

LABine, Alfred C., M.D., 8-259 General Motors Bldg., Detroit, Mich. ('28).

LAMAR, Norvelle C., M.D., I 49 E. 73rd St., New York, N. Y. (1) ('3o).

Langrord, William S., M.D., Babies Hospital, 167 th St. and Broadway, New York, N. Y. ('36).

Langner, Helen P., M.D., Vassar College, Poughkeepsie, N. Y. ('29).

Larson, John A., M.D., Psychopathic Clinic, Recorder's Court, Detroit, Mich. ('31).

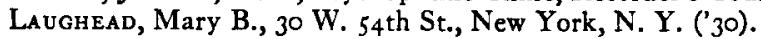

LaVallee, Joseph, M.D., 423 Commercial Square, Cincinnati, O. ('39).

Lawton, George, Ph.D., 35 W. 82nd St., New York, N. Y. ('35).

Leonard, Christina M., M.D., 30 W. $54^{\text {th }}$ St., New York, N. Y. (2) ('24).

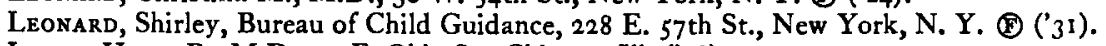

Levey, Harry B., M.D., 43 E. Ohio St., Chicago, Ill. ('38).

Levy, David M., M.D., I 36 E. 57 th St., New York, N. Y. (1) (C).

Lewin, Bertram D., M.D., 25 Fifth Ave., New York, N. Y. ('31).

LEwIs, Margaret, 2450 Overlook Rd., Cleveland, O. ('35).

Libzey, Betsey, 3 I I S. Juniper St., Philadelphia, Pa. (' $3 \mathrm{I}$ ).

Lippman, Hyman S., M.D., Amherst H. Wilder Ch.Guidance Clin., 279 RiceSt., St. Paul, Minn. (') ('28). 
Liss, Edward, M.D., 130 E. 39th St., New York, N. Y. (1) ('32).

Lithauer, Donah B., 520 W. I Ioth St., New York, N. Y. ('32).

Litrte, Harry M., M.D., Pittsburgh Child Guidance Center, 3604 Victoria St., Pittsburgh, Pa. ('35).

Lloyd, Wilma F., West Redding, Conn. ('31).

Lorand, Sandor, M.D., 115 E. 86th St., New York, N. Y. (5) ('33).

LotT, George M., M.D., 840r Chapin Pkwy, Jamaica, N. Y. ('33).

Low REY, Lawson G., M.D., 28 W. $54^{\text {th }}$ St., New York, N. Y. (P) (C).

Lowry, Fern, 34 E. 22nd St., New York, N. Y. ('37).

Luckey, Bertha M., Ph.D., Board of Education, Cleveland, O. (ㄱ) ('31).

Luenrs, Leslie E., M.D., I 4 Washington Square N., New York, N. Y. ('29).

Lurie, Louis A., M.D., Doctors Bldg., Cincinnati, O. (F) ('26).

Lurie, Olga Rubinow, Ph.D., 5463 Cornell Ave., Chicago, Ill. ('39).

Lyman, Richard, M.D., Johns Hopkins Hospital, Baltimore, Md. ('34).

MacDonald, Martha W., M.D., Guidance Center, Hutchinson Mem. Bldg., New Orleans, La. (F) ('33).

MacFarlane, Jean W., Ph.D., I 542 LaLoma Ave., Berkeley, Calif. ('39).

McBee, Marian, 405 E. 54th St., New York, N. Y. ('39).

McClure, Jeanette E., Children's Center, 3743 Brush St., Detroit, Mich. ('32).

McClure, Mrs. Robert, Pittsburgh Child Guidance Center, 3604 Victoria St., Pittsburgh, Pa. ('31).

McCord, Clinton P., M.D., 54 Willett St., Albany, N. Y. (D) ('26).

McNiel, Edwin E., M.D., Queens Hospital, Honolulu, T. H. ('37).

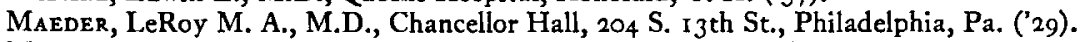

Malcove, Lillian, M.D., 245 E. $72 n$ St., New York, N. Y. ('3I).

Markey, Oscar B., M.D., 7016 Euclid Ave., Cleveland, O. (E) ('28).

Marshall, Berry C., M.D., 419 Hawthorn Rd., Roland Park, Baltimore, Md. ('33).

Marshall, Helen, R.R.I., Box 374 Palo Alto, Cal. ('34).

Martin, A. Leila, Ph.D., Child Study Dept., Bd. of Ed., I 3 Fitzhugh St., S. Rochester, N. Y. (2) ('26).

Mayer, Edward E., M.D., Medical Bldg., Pittsburgh, Pa. ('34).

Mellor, Ruth, Mental Hygiene Clinic, 6ro S. Floyd St., Louisville, Ky. ('39).

Meltzer, H., Ph.D., 4510 Maryland Ave., St. Louis, Mo. (2) ('31).

Menarer, Esther, Ph.D., 35 W. 82nd St., New York, N. Y. ('39).

Menninger, Karl A., M.D., Menninger Clinic, 3617 W. 6th Ave., Topeka, Kans. (A) (C).

Menninger, William C., M.D., Menninger Clinic, 36r7 W. 6th Ave., Topeka, Kans. ('35).

MEYER, Adolf, M.D., Johns Hopkins Hospital, Baltimore, Md. (D) ('34).

Michaels, Joseph J., M.D., 37 Marlborough St., Boston, Mass. (' 38 ).

Mrtchell, William T. B., M.D., McGill University, Montreal, Canada. (F) ('26).

MoHR, Mrs. Esther J., 5539 Blackstone Ave., Chicago, Ill. ('32).

Мон,, George J., M.D., 43 E. Ohio St., Chicago, III. (2) ('26).

Montague, Helen, M.D., 27 W. gth St., New York, N. Y. (1) ('24).

Moore, Madeline U., 5o E. 1oth St., New York, N. Y. ('33).

Morsh, Mrs. Edith Symmes, 5570 Chancellor Blvd., Vancouver, B. C., Canada. ('28).

Movlton, Bryant E., M.D., I 8 Symmes Rd., Winchester, Mass. ('31).

Moulton, Phyllis, 315 E. 68 th St., New York, N. Y. (' 31 ).

Moyle, Henry B., M.D. (Tor.), Hartley-Salmon Clinic, 488 Main St., Hartford, Conn. (F) ('29).

Murphey, Bradford J., M.D., Child Guidance Clinic, 64 W. Ross St., Wilkes-Barre, Pa. (F) ('29).

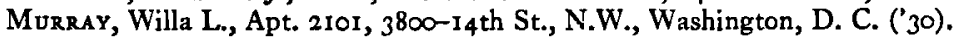

Muskat, Caroline J., c/o Dr. Muskat, Marietta, 0 . ('32).

Nelson, William, M.D., 5449 Delmar Blvd., St. Louis, Mo. ('29).

Neumans, Frederika, I 9 W. Ioth St., New York, N. Y. (if ('3I).

Neweli, H. Whitman, M.D., Ment. Hyg. Soc. Md., 60I W. Lombard St., Baltimore, Md. (7) ('30).

Oberndorf, Clarence P., M.D., 1 I 2 W. 59th St., New York, N. Y. (F) ('31).

O'Brien, Frank J., M.D., Bureau of Child Guidance, 228 E. 57th St., New York, N. Y. (2) ('25).

O'Shea, Harriet, Ph.D., Purdue University, Lafayette, Ind. ('39).

Olson, Elma, Family Welfare Association, I 563 Oak Ave., Evanston, Ill. (i) ('29).

Olson, Willard C., Ph.D., School of Education, University of Michigan, Ann Arbor, Mich. (2) ('29).

Orger, Samuel Z., M.D., 667 Madison Ave., New York, N. Y. (D) ('29).

Parker, Z. Rita, M.D., I 5 E. 6rst St., New York, N. Y. ('28).

Patry, Frederick L., M.D., 21 4 State St., Albany, N. Y. (i) ('32). 
Paynter, Richard H., Ph.D., 395 Grand Ave., Brooklyn, N. Y. (F) ('30).

Pearson, Gerald H. J., M.D., Pennsylvania Hospital, i I N. 49th St., Philadelphia, Pa. (-) ('3o).

Peppard, S, Harcourt, M.D., 35 W. 9th St., New York, N. Y. (E) ('3i).

Perrans, Nellie, Ph.D., 834 Trombley Rd., Grosse Point, Mich. ('25).

PerRY, Doris E., Io Adelaide St., Portland, Me. ('30).

Pratr, George K., M.D., 80 Myrtle Ave., Westport, Conn. (D) ('27).

Preston, George H., M.D., 330 N. Charles St., Baltimore, Md. (1) ('29).

PrItchetT, Henry L., Ph.D., Southern Methodist University, Dallas, Tex. ('3I).

Putnam, Marian C., M.D., II 2 Revere St., Boston, Mass. (F) ('32).

Quinlan, Gertrude, 130 E. 57 th St., New York, N. Y. ('35).

Rademacher, E. S., M.D., 442 Temple St., New Haven, Conn. (1) ('27).

Ranck, Katherine H., I I00 N. Dearborn Pkwy., Chicago, Ill. ('39).

Raphael, Theophile, M.D., Univ. Health Service, Univ. of Michigan, Ann Arbor, Mich. (F) ('26).

Reeve, George H., M.D., 10456 Carnegie Ave., Cleveland, O. (1) ('26).

Reichen berG, Wally, Ph.D., Dept. of Psychology, Fordham University, Bronx, N. Y. ('38).

Reynolds, Bertha C., 3947-48th St., Long Island City, N. Y. (10) ('30).

Rich, Gilbert J., M.D., Mental Hygiene Clinic, 515 Public Safety Bldg., Milwaukee, Wis. (29) ('29).

Richie, Richard F., M.D., I6ro S. 24th St., Lincoln, Neb. ('39).

Richmond, Winifred, Ph.D., St. Elizabeths Hospital, Washington, D. C. ('36).

Rivlın, Harry N., Ph.D., City College, Convent Ave. and I 39th St., New York, N. Y. ('39).

RoвiE, Theodore R., M.D., zo Norman Rd., Upper Montclair, N. J. ('3o).

Robinson, Bruce B., M.D., Dept. of Child Guidance, 31 Green St., Newark, N. J. (E) ('29).

Roвinson, Lindsay Ellsworth, M.D., 332 Park Ave., Newark, N. J. ('37).

Rockwell, Alice J., Ph.D., Ment. Hygiene Soc. Maryland, 6or W. Lombard St., Baltimore, Md. ("33).

Rogers, Carl R., Ph.D., Rochester Guidance Center, 3I Gibbs St., Rochester, N. Y. (D) ('34).

Roor, June A., 44 Quarropas St., White Plains, N. Y. ('3I).

Rosenblum, Deborah F., Jewish Board of Guardians, 228 E. 19th St., New York, N. Y. (D) ('3r).

Rosenheim, Frederick, M.D., Judge Baker Guidance Center, $38 \frac{1}{2}$ Beacon St., Boston, Mass. ('39).

Rosenzweig, Leonard, M.D., Warren State Hospital, Warren, Pa. ('39).

Rosenzweig, Saul, Ph.D., Worcester State Hospital, Worchester, Mass. (ii) ('35).

Ross, Helen, II 5 I E. 56th St., Chicago, Ill. ('39).

Ross, Mrs. Mildred Midnight, 4410 Franklin Ave., Cleveland, O. ('37).

Rossman, Max, M.D., Allentown State Hospital, Allentown, Pa. ('38).

Roтн, William F., Jr., M.D., Williamson County Child Guidance Study, Franklin, Tenn. ('35).

Rugcles, Arthur H., M.D., 305 Blackstone Blvd., Providence, R. I. ('29).

Scha piro, Joseph, M.D., Bureau of Child Guidance, 712 Palisade Ave., Union City, N. J. ('35).

Schroeder, Paul L., M.D., Finst. for Juvenile Research, 907 S. Wolcott Ave., Chicago, Ill. (1) ('30).

Schumacher, Henry C., M.D., Child Guidance Clinic, 2525 Euclid Ave., Cleveland, O. (2) ('26).

Schwartz, Louis A., M.D., 1079 Fisher Bldg., Detroit, Mich. ('31).

Schwarzenberg, Edith A., N. Y. School of Social Work, I 22 E. 22nd St., New York, N. Y. ('3I).

Scoville, Mildred C., The Commonwealth Fund, 4 I E. 57 St., New York, N. Y. (2) ('26).

See berg, Elizabeth, Ph.D., 147 Mason St., Cincinnat, O. ('28).

Selling, Lowell S., M.D., I6Ig6 Cherrylawn Ave., Detroit, Mich. (if) ('30).

Senior, Rose D., M.D., 5612 Kenwood Ave., Chicago, Ill. ('35).

SHAKow, David, Worcester State Hospital, Worcester, Mass. (7) ('30).

Siewers, Albert B., M.D., Medical Arts Bldg., Syracuse, N. Y. (1) ('29).

Silverman, Baruch, M.D., 53I Pine Ave., W., Montreal, Canada. (') ('31).

Skinner, Anna M., M.D., 4 Bates Rd., Watertown, Mass. ('29).

Slawson, John, Ph.D., Jewish Board of Guardians, 228 E., I gth St., New York, N. Y. (1) ('36).

Sloane, Paul, M.D., 269 S. 19th St., Philadelphia, Pa. ('37).

SMAlley, Ruth E., Smith College School for Social Work, Northampton, Mass. ('36).

SMIth, Barry C., The Commonwealth Fund, 4 I E. 57th St., New York, N. Y. (H) ('3o).

Sмiтн, Groves Blake, M.D., Beverly Farms, Inc., Godfrey, Ill. (i) ('3o).

Sozomon, Joseph C., M.D., Ment. Hygiene Soc. Maryland, 60I W. Lombard St., Baltimore, Md. ('38).

Sommer, Conrad, M.D., Illinois Society for Mental Hygiene, 203 N. Wabash Ave., Chicago, Ill. ('38).

Spencer, W. Douglas, Ph.D., Apt. I2B, 445 W. 23rd St., New York, N. Y. ('32).

SPEYER, Helen, 70 E. 77th St., New York, N. Y. ('37).

Steiner, Mrs. Lee Rabinowitz, 25 W. 85th St., New York, N. Y. ('38).

Stephenson, Charles W., M.D., I79 Allyn St., Hartford, Conn. ('32). 
Stevenson, George S., M.D., Nat. Comm. for Ment.Hygiene, 50 W. 50 th St., New York, N.Y (․(C). Stoy, Edward G., Ph.D., 68 Post St., San Francisco, Cal. ('3I).

Stranahan, G. Marion, M.D., 6i E. 86th St., New York, N. Y. (F) ('3I).

Strecker, Edward A., M.D., Pennsylvania Hospital, i i N N. 49 th St., Philadelphia, Pa. (1 ('24).

Sullivan, Harry Stack, M.D., 158 E. 64th St., New York, N. Y. (1) ('30).

Sumner, Mary C., Family Society, 484 Orange St., New Haven, Conn. ('26).

Svendsen, Margaret T., Institute for Juvenile Research, $907 \mathrm{~S}$. Wolcott Ave., Chicago, Ill. ('34).

Symonds, Percival M., Ph.D., Teachers College, Columbia University, New York, N. Y. ('37).

Swirt, Sarah H., io35 Fifth Ave., New York, N. Y. (F) ("26).

TAFt, Jessie, Ph.D., 3I I S. Juniper St., Philadelphia, Pa. (F) ('25).

Tallman, Frank F., M.D., Rockland State Hospital, Orangeburg, N. Y. ('38).

Taussig, Helen P., 235 E. 22nd St., New York, N. Y. ('3I).

Teagarden, Florence M., Ph.D., University of Pittsburgh, Pittsburgh, Pa. ('37).

Thompson, Lloyd J., M.D., New Haven Hospital, New Haven, Conn. (E) ('29).

Thurrott, John C., M.D., 965 Fifth Ave., New York, N. Y. (B) ('3I).

Tiebout, Harry M., M.D., 215 Milbank Ave., Greenwich, Conn. (F) ('28).

Tolman, Ruth Sherman, Ph.D., 345 S. Michigan Ave., Pasadena, Cal. ('39).

Towle, Charlotte, 574I Kenwood Ave., Chicago, Ill. (B) ('38).

Tracy, Anne Belle, School of Applied Social Sciences, Western Reserve University, 2117 Adelbert Rd., Cleveland, O. (F) (' 30 ).

Trurt, Ralph P., M.D., Ment. Hygiene Soc. Maryland, 6oI W. Lombard St. Baltimore, Md. (') ('3o).

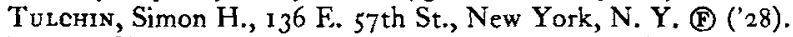

Uhler, Claude, M.D., State Mental Hygiene and Child Guidance Clinic, Farnhurst, Del. ('39).

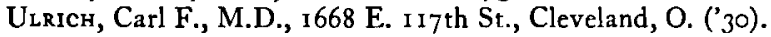

Van Hyning, Mrs. Mary Whitehead, Dallas, Pa. ('30).

Vedder, Mrs. Sarah V. D., Rock Rimmon Rd., Stamford, Conn. ('3i).

Wagner, Carl P., M.D., 25 Marlborough St., Portland, Conn. ('34).

Watson, Goodwin, Ph.D., Teachers College, Columbia University, New York, N. Y. ('33).

Watson, Maud E., Ph.D., 2240 W. Grand Blvd., Detroit, Mich. ('34).

Webber, Alice J., 310 W. 97 th St., New York, N. Y. ('3I).

Wechsler, David, Ph.D., Bellevue Psychiatric Hospital, 400 E. 3oth St., New York, N. Y. ('37).

Weill, Blanche C., Ed.D., 470 W. 24th St., New York, N. Y. ('35).

Wells, Frederick L., Ph.D., 13 Holyoke St., Cambridge, Mass. ('39).

We lsch, Exie E., M.D., Dept. Public Welfare, I4I S. Meridian St., Indianapolis, Ind. ('39).

Westrurgh, Edward M., Ph.D., The Institute, in I N. 49th St., Philadelphia, Pa. ('39).

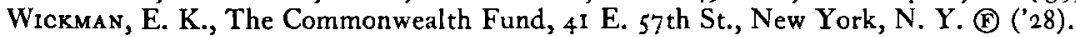

Wickman, Mrs. K. M., Pediatric-Psychiatric Clinic, I67th St. and Broadway, New York, N. Y. (i) ('29).

Wilderson, Myrna, 2450 Overlook Rd., Cleveland, O. ('37).

Wile, Ira S., M.D., 264 W. 73rd St., New York, N. Y. (P) ('26).

Wiltshire, Helen, 225 N. Jefferson St., Dayton, O. (' 38 ).

Winsor, Max, M.D., Bureau Child Guidance, P.S. 24, 22 E. I28th St., New York, N. Y. ('39).

Witmer, Helen Leland, Ph.D., 308 E. 15 th St., New York, N. Y. (A) ('34).

Witmer, Prof. Lightner, University of Pennsylvania, Philadelphia, Pa. ('25).

Wolfr, Ernst, M.D., 450 Sutter St., San Francisco, Cal. ('39).

Woltmann, Adolf G., 108 E. 96th St., New York, N. Y. ('39).

Wood, Louise, Judge Baker Guidance Center, $38 \frac{1}{2}$ Beacon St., Boston, Mass. (' 38 ).

Woolley, Lawrence F., M.D., 6 Dixie Dr., Towson, Md. ('32).

Young, Paul C., Ph.D., Louisana State University, Baton Rouge, La. ('37).

Zachry, Caroline B., Ph.D., 17 E. 96th St., New York, N. Y. (P) ('30).

Zilboorg, Gregory, M.D., 14 E. 75 th St., New York, N. Y. (E) ('32). 